\title{
Optimized calculation of high strain rate stress-strain curves
}

\author{
P. Verleysen \& J. Degrieck \\ Ghent University, Faculty of Engineering, \\ Department of mechanical Construction and Production, Belgium
}

\begin{abstract}
The knowledge and understanding of high strain rate material behaviour have become increasingly important. For the dynamic characterization of materials very often split Hopkinson bar experiments are used. However, as will be shown, the specimen geometry and other parameters related with the actual test setup and execution have a distinct influence on the obtained stress-strain curves. In this contribution a technique is presented which iteratively corrects the stressstrain curve extracted from an experiment by combination of numerical simulations and experimental results. A major advantage of the technique presented here is that the stress-strain curve extracted from the experiment itself is corrected; no prior assumptions on the material behaviour have to be made.
\end{abstract}

Keywords: high strain rate, split Hopkinson bar, Kolsky apparatus, stress-strain curve, optimization.

\section{Introduction}

In numerous applications materials are subjected to high strain rate loading: fast forming processes, magnetic pulse or explosive welding techniques, vehicle crashes, etc. In order to understand and/or model these processes, knowledge of the strain rate dependent mechanical properties of the materials involved is crucial. In the strain rate range of 500 to 2000/s very often Split Hopkinson Bar (SHB) setups are used to subject materials to a high strain rate compression or tensile load. From measurements during a SHB experiment the dynamic stressstrain curve in the material can be calculated.

However, from experiments, it is clear that the influence of the specimen geometry, or more general of the experimental setup, on the observed behaviour 
cannot be neglected: the stress-strain curve extracted from the recorded signals during a SHB experiment using the classical equations is highly specimen geometry dependent. The observed mechanical behaviour is a combination of structural and material response. Consequently, the comparison with experimental results from static tests, other dynamic tests or other laboratories is most often impossible. Also in static experiments the specimen geometry can have an influence on the obtained results. The situation is more critical for high strain rate experiments however, because the short duration of the experiment, the small size of the specimens and wave propagation effects, make a straightforward and accurate measurement of forces and deformations much more difficult.

Evidently, it is of utmost importance that the obtained stress and strain histories are an accurate representation of the real material behaviour. In order to obtain a stress-strain curve that represents the actual material behaviour, a technique is developed which iteratively corrects the stress-strain curve extracted from an experiment by combination of numerical simulations and experimental results. The technique is illustrated by SHB tensile experiments on a TRIP-steel sheet.

\section{SHB tensile experiment}

\subsection{Classic calculation of strain, strain rate and stress}

During a SHB tensile experiment a material sample is fixed between two Hopkinson bars: an input bar and an output bar (fig. 1). The specimen is subjected to a high strain rate tensile load through the interaction of a tensile wave, generated at the free end of the input bar, with the specimen. The incident wave is partly reflected and partly transmitted by the specimen. The strain histories $\varepsilon_{\mathrm{i}}(\mathrm{t}), \varepsilon_{\mathrm{r}}(\mathrm{t})$ and $\varepsilon_{\mathrm{t}}(\mathrm{t})$ corresponding to respectively the incident, reflected and transmitted wave are measured at well chosen points on the Hopkinson bars. After shifting the waves towards the specimen/bar interfaces, the stress, the strain and the strain rate in the specimen can be calculated using the following expressions [1]:

$$
\begin{gathered}
\sigma(t)=\frac{A_{b} E_{b}}{A_{s}} \varepsilon_{t}(t) \\
\dot{\varepsilon}(t)=\frac{V_{o b}-V_{i b}}{L_{s}}=-\frac{2 C_{b}}{L_{s}} \varepsilon_{r}(t) \quad \varepsilon(t)=\frac{U_{o b}-U_{i b}}{L_{s}}=-\frac{2 C_{b}}{L_{s}} \int_{0}^{t} \varepsilon_{r}(\tau) d \tau
\end{gathered}
$$

with $E_{b}$ the modulus of elasticity of the Hopkinson bars, $A_{s}$ and $A_{b}$ the cross section area of the specimen and of the Hopkinson bars respectively, $C_{b}$ the velocity of propagation of longitudinal waves in the Hopkinson bars and $\mathrm{L}_{\mathrm{s}}$ the gage length of the specimen. $U_{i b}$ and $U_{o b}$ are the displacements of the interface between the specimen and, respectively, the input bar and the output bar; $\mathrm{V}_{\mathrm{ib}}$ and $\mathrm{V}_{\mathrm{ob}}$ are the corresponding velocities.

The equations above are based on the assumption that in the specimen a quasi-static equilibrium is established. Inertial forces acting on the specimen are 
omitted; the tensile forces at the interfaces of the specimen with the input bar and output bar, and consequently in each transverse section of the specimen, are equal. In the zone of the specimen where a constant cross-section exists stresses, as well as strains and strain rates, are considered to be homogeneous. Moreover, stresses are assumed to be uniaxial in this zone. Finally, the deformation of the transition zones, where the section of the specimen is changing, is neglected.

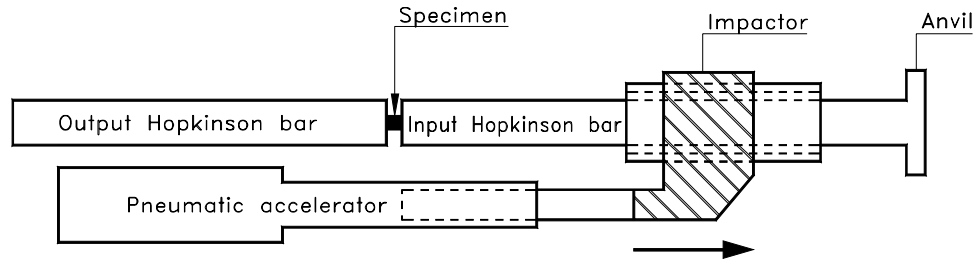

Figure 1: $\quad$ Experimental setup of a typical split Hopkinson tensile bar device.

\subsection{Specimen geometry}

A schematic representation of the specimen geometry is given in fig. 2. It consists of a central zone with a constant width and a certain length, and, on both ends of the central zone, transition zones where the width gradually increases following a circular curve. The zones of the specimen shaded in fig. 2 are needed to glue the specimen in slits at the ends of the Hopkinson bars.

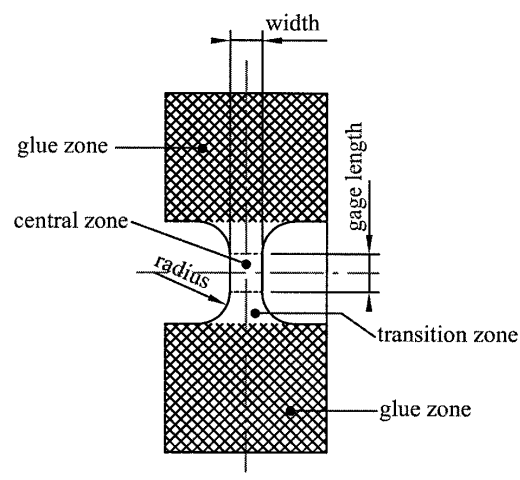

Figure 2: $\quad$ Schematic representation of the specimen geometry.

In order to study the influence of the specimen geometry on the obtained results, 7 geometries are defined. The dimensions of a reference geometry are established based on geometries described in literature: the length of the central zone is $5 \mathrm{~mm}$, the width of this zone is $4 \mathrm{~mm}$, and the radius of the transition zones is $2 \mathrm{~mm}$. Starting from that reference geometry, six additional geometries are determined by varying the radius of the transition zones, the length and the width of the central zones. Each time only one parameter is changed while the 
other two remain constant. Accordingly, the influence of the radius $\mathrm{R}$ of the transition zones (geometry $3: \mathrm{R}=1 \mathrm{~mm}$ and $4: \mathrm{R}=4 \mathrm{~mm}$ ), the length 1 (geometry $5: 1=10 \mathrm{~mm}$ and $6: 1=3.33 \mathrm{~mm}$ ) and the width $\mathrm{w}$ of the cross-section (geometry $2: \mathrm{w}=6 \mathrm{~mm}$ and $7: \mathrm{w}=2 \mathrm{~mm}$ ) can be studied.

In fig. 3 stress-strain curves corresponding with a strain rate of approximately $825 / \mathrm{s}$ are represented for the seven geometries. These curves are calculated from the signals measured by the strain gages on the Hopkinson bars using the above equations. As can be seen, the specimen geometry has a pronounced effect on the stress-strain curves. Particularly, the influence on the specimen deformation is very pronounced. Indeed, for the experiments presented in this figure, the uniform elongation ranges from 0.234 (geometry 5) to 0.367 (geometry 6), i.e. an increase of $57 \%$.

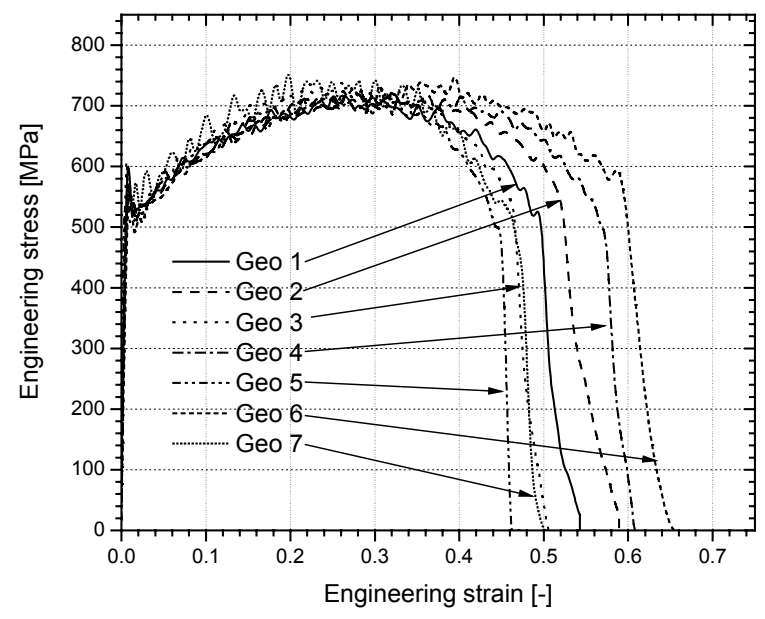

Figure 3: $\quad$ Stress-strain curves for the seven geometries derived from strain gage measurements on the bars using the classical processing technique (strain rate approximately $825 / \mathrm{s}$ ).

\section{Finite element model}

The numerical simulations were performed using the finite element program ABAQUS (from ABAQUS, Inc.). The finite element model was created with ABAQUS/CAE. Since we wanted to study the precise dynamical phenomena in the specimen, ABAQUS/Explicit code was used for the calculations.

To correctly take into account inertia and wave propagation phenomena, the interaction of a real wave with the specimen is modelled. The model comprises the test specimen and parts of both Hopkinson bars long enough to have no interference of reflected stress waves with the specimen during the time period of interest. Bars of $2 \mathrm{~m}$ length were sufficient. Because of the symmetry only one quarter of the cross-section was modelled, and symmetry conditions imposed. The different specimen geometries and the Hopkinson bars were created as 
separate parts in Abaqus/CAE, and subsequently assembled. A test specimen was connected to the Hopkinson bars by a tied interface on the side surfaces only. The butt of the specimen was not tied to the Hopkinson bar, as it is unlikely that the glue can transmit the high stresses that would result. Loading on the model is applied as a stress wave on the end of the input Hopkinson bar. The stress wave used was derived from strain gauge readings on the input bar of the incident wave during a real test. For the specimens a classical metal, elasto-plasticity model with isotropic hardening was used. For the study of the influence of the specimen geometry tabulated data were entered, corresponding to the stressstrain relationship established during a static tensile experiment on a Al-TRIP steel [2].

A finite element mesh was generated with 4 elements through the (half) thickness of the specimen. The mesh is finer in the smaller section of the specimen, where elements are approximately cubes. The Hopkinson bars were meshed quite coarser, since they are not of interest for our study and only serve to transmit the loading on the specimen. The element type used is C3D8R, an eight node linear brick type element using reduced integration with hourglass control. This is the obvious choice for modelling 3D solids under highly dynamic conditions. The element mesh for the specimen in the figure comprises 14060 nodes and 10528 elements. Each of the Hopkinson bars accounted for approximately 34005 nodes and 25957 elements. Total runtime including data check and full analysis was about 6 hours.

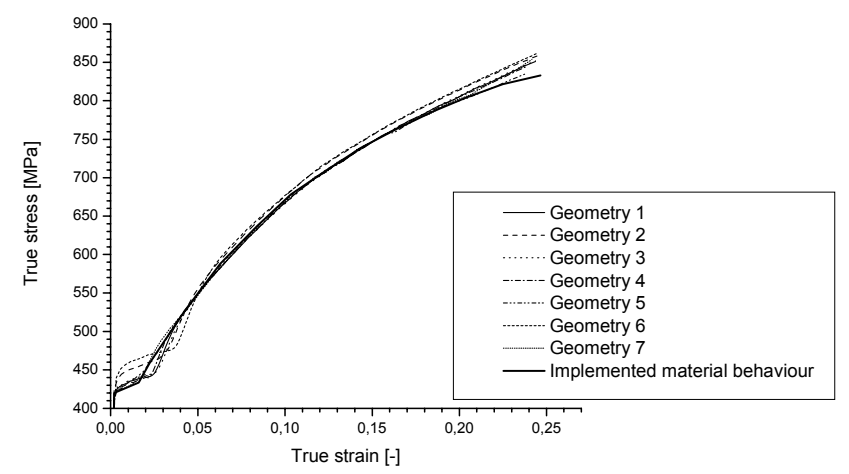

Figure 4: Stress-strain curve in the centre of the specimen for the seven geometries, and the material law used for the simulations.

The simulated axial stress-strain curves in the centre are represented in fig. 4 for all geometries, together with the implemented law. Here again, clear deviations between the material law and the curves for the different geometries are obvious during all phases of the deformation. The simulations learned that commonly neglected non-axial stresses have a major influence on both the specimen response and the accuracy of the classical method used to extract strain and strain rate from the waves recorded during an experiment. These non-axial 
stresses are dependent on both the specimen geometry and, diameter and material of the Hopkinson bars.

Also neglecting the deformation of the transition zones gives rise to serious errors in the strain history calculated from a SHB experiment. In fig. 5 the relative contribution of the transition zones to the total deformation is represented for all geometries. As can be seen, the contribution from the transition zones is not negligible. Since, the deformation of the transition zones is added to the deformation of the central zone, the classically obtained strain values are an overestimation for the actual strain in the central zone of the specimen.

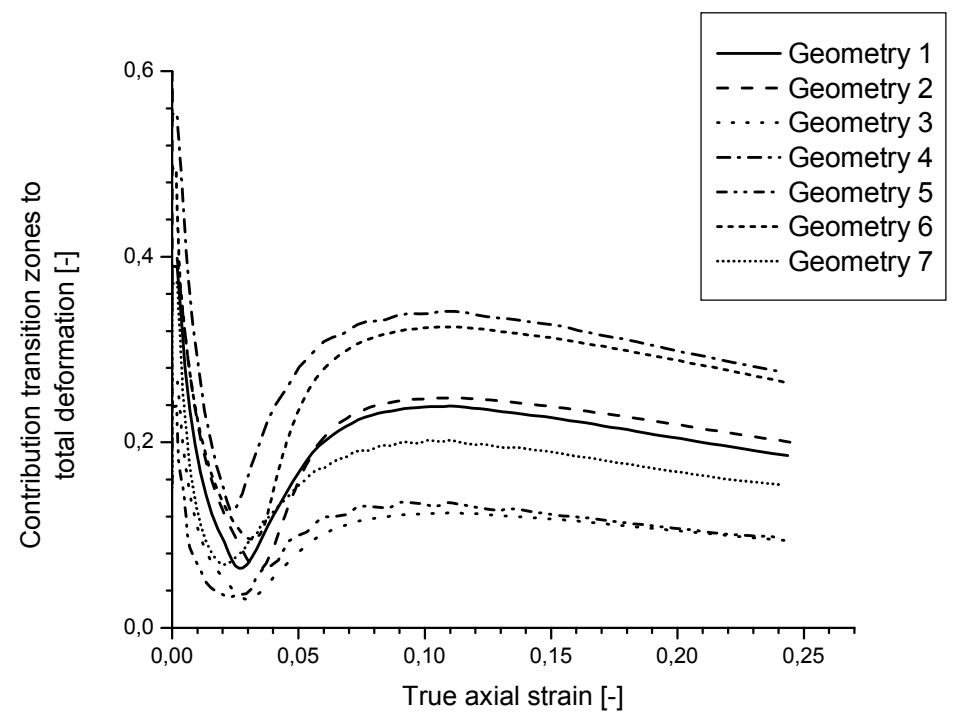

Figure 5: Relative contribution of the deformation of the transition zones to the total specimen deformation.

\section{Optimization technique}

An important conclusion from the experimental and numerical results presented in paragraphs 2 and 3 is that the specimen geometry has a distinct influence on the results obtained with a SHB test. For the 7 geometries considered here the small radius geometry 3 gives the best results, i.e. the extracted behaviour is the closest to the actual material behaviour around the centre of the specimen. Unfortunately, this conclusion is only valid for the material and the strain rate considered here. For a more brittle material for instance the stress concentration caused by the sharp radius can give rise to an early failure of the specimen at the shoulder. For materials with another hardening behaviour, the contribution of the 
deformation of the transition zones to the total deformation will change, and thus the classical deformation calculation can yield more accurate deformation values for other geometries than geometry 3. Moreover for materials with a high strain rate sensitivity, the variation of the transitions zone deformation contribution with the strain rate can also be non-negligible and thus the optimal geometry is strain rate dependent. Consequently, one optimal specimen geometry does not exist and enhancing the results of high strain rate experiments by optimizing the specimen geometry is elaborate since it has to be repeated for each material, and eventually each strain rate, studied

To enhance the accuracy of high strain rate experiments we opted therefore for a two-step approach. In a first step we choose a geometry that guarantees quasi-static equilibrium from the early stages of loading, but more important low axial stresses. The first constraint mainly imposes limits to the length of the specimen, for the second a minimum length to width ratio of the central zone of the specimen is required. The deformation of the transition zones is not a parameter in the geometry selection process. In a second step the obtained experimental results are iteratively enhanced. Using the finite element model described in paragraph 3 , the alternative procedure for the interpretation of the test results consists of the following steps (fig. 6):

Step 1. From the SHB experiment the histories of the relative displacement of the specimen/bar interfaces, the stress and strain curve are calculated using the equations of paragraph 2 . The obtained experimental stressstrain curve is implemented as material model for the specimen behaviour in the FE model of the SHB setup described in paragraph 3.

Step 2. The SHB-experiment is simulated with the FE model.

Step 3. From the simulation results, the histories of the axial stress in the central section of the specimen and the relative displacement of the specimen/bar interfaces are extracted.

Step 4. The simulated stress versus relative displacement of the specimen ends curve is compared with the experimentally obtained curve. When the agreement is insufficient, step 2 and 3 have to be repeated using an improved stress-strain curve for the specimen material behaviour. The improved stressstrain curve is obtained by correcting each strain value of the stress-strain relation used in the previous iteration step using the ratio of the experimental to the simulated relative displacement corresponding with the same stress level.

Note that with this technique, no assumptions have to be made on the specimen behaviour. Here, the optimization is used for a SHB tensile experiment; however, without significant changes the principle of the iterative correction procedure can also be used for other experiments.

To study the accuracy of the optimization technique, an experiment on a material with an assumed material behaviour was simulated. From the simulation results, a stress-strain curve was calculated using the equations given in paragraph 2.1. This stress-strain curve was used for the first iteration and subsequently iteratively corrected using the technique described above.

In figure 7 the assumed material behaviour, the simulated-experimental material behaviour and the result of the first correction are presented. As can be 


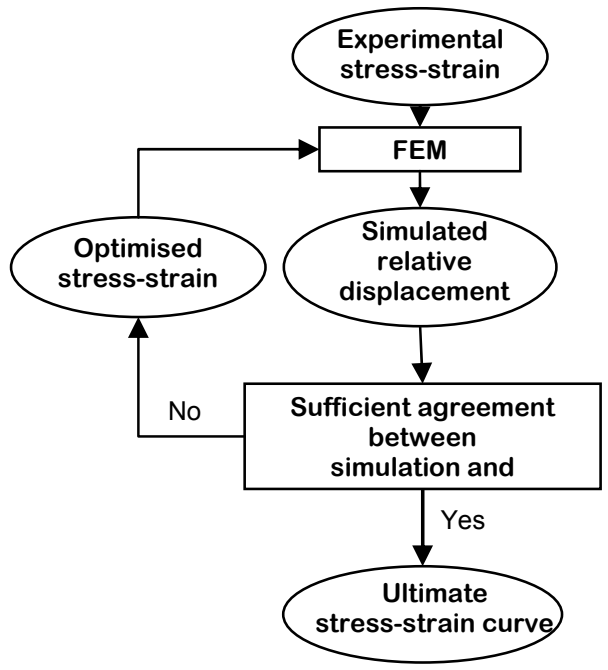

Figure 6: Iterative correction procedure for the results of SHB tests.

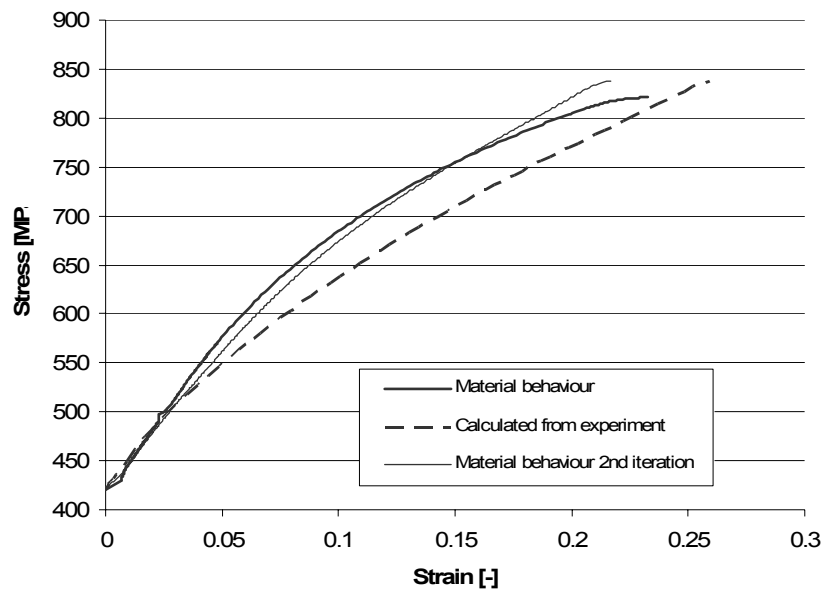

Figure 7: $\quad$ Stress-strain curve extracted from a SHB tensile experiment (and used for the first iteration), material law used for the second iteration and actual material stress-strain relation.

seen in this figure, the stress-strain curve used for the second iteration step is already very close to the actual material law. The geometry considered here is geometry 6 , the geometry where due to its low length/width ratio the highest non-axial stresses occur. It can consequently be concluded that even when the starting geometry is not optimal, fast convergence is obtained and the finally obtained results are satisfactory. 


\section{Conclusion}

Although a SHB experiment is assumed to yield the dynamic material behaviour, it is shown that parameters related with the actual test setup, such as the specimen geometry, have a significant influence on the obtained results. As shown by numerical simulations, the main reason for this is that the assumptions on which the calculation of the stress-strain curve is based are not fulfilled. In this contribution a technique is presented that iteratively corrects the experimentally obtained stress-strain curve to finally obtain a curve representing the actual material behaviour. Although the technique was illustrated by a SHB tensile experiment, the combination of experiments and numerical simulations to iteratively improve the experimental results can be useful wherever uncertainties exist on the assumptions used to extract the results.

\section{References}

[1] Kolsky H. An investigation of the mechanical properties of materials at very high rates of loading. Proc Phys Soc Lond Sec B 1949;62:676-700.

[2] Verleysen P, Van Slycken J, Degrieck J, De Cooman B, Samek L. Crashworthiness of low alloy TRIP steel. Proc Int Conf on advanced high strength sheet steel for automotive applications 2004. Colorado. 